\title{
Organizaciones no gubernamentales y violencia de género: Caso Nuevo León, México
}

\author{
Non-Gubernamental Organizations \\ and Gender Violence: Case Nuevo Leon, Mexico
}

\author{
Pedro César Cantú-Martínez \\ Universidad Autónoma de Nuevo León, México \\ pedro.cantum@uanl.mx \\ https://orcid.org/0000-0001-8924-5343
}

Recibido: 26-04-2021 Revisado: 11-06-2021 Aceptado: 30-06-2021 Publicado: 01-09-2021

\section{Resumen}

Este artículo plantea el análisis de la actuación de las organizaciones no gubernamentales, que brindan apoyo solidario y desinteresado ante la problemática subsistente de violencia en contra de la mujer. En México, este problema social ha sido difícil de atender, ya que cuenta con arraigo social como cultural. El escenario se presenta en el marco nacional y adicionalmente se observa la estructuración gubernamental y la organización no gubernamental mediante la sociedad civil que acoge esta eventualidad social en Nuevo León. Se empleó una metodología de carácter descriptivo y de representación cualitativa, se accedió a informes y documentos, lo que permitió acotar el objeto de estudio. El escenario de Nuevo León recrea la situación social y de violencia que en México subsiste. Para Nuevo León, que aglomera el $4.3 \%$ de las mujeres del país, la violencia persiste en el 59.3\% de ellas. Principalmente esta fue perpetrada de manera comunitaria, siguiéndole la impuesta por su pareja. La estructura gubernamental cuenta con un tejido administrativo que presta servicios jurídicos y asistenciales. Sin embargo, la sociedad civil se ha organizado para atender esta contingencia, y en Nuevo León existe una red de organizaciones las cuales $4.87 \%$ están relacionadas con la atención de la mujer en estos casos. Las mismas cumplen funciones asistenciales, de derechos humanos, de autocuidado, de educación y orientación, de servicios de salud mental, de formación de capacidades y reinserción social.

\section{Palabras clave}

Sociedad civil, organizaciones no gubernamentales, atención, violencia, género, mujeres.

Forma sugerida de citar: Cantú-Martínez, P.C. (2021). Organizaciones No Gubernamentales y violencia de género: Caso Nuevo León, México. Universitas-21,35-XXI, pp. 151-165. https://doi.org/10.17163/uni.n35.2021.07 


\begin{abstract}
This paper addresses the role of non-governmental organizations, providing solidarity support and disinterested in the problem of violence against women. In Mexico this social problem has been difficult to solve, because it has social and cultural roots. The stage is presented in the national framework and we also see government structuring such as the non-governmental organization through civil society that hosts this social eventuality in Nuevo Leon. A descriptive methodology was used and qualitative representation, reports and documents were accessed, that allowed us to delimit the object of study. Nuevo León's scenario repeats social situation and violence that persists in Mexico. Nuevo Leon has $4.3 \%$ of the country's women, violence persists in $59.3 \%$ of them. First cause was in a communal way, following the one imposed by his partner. Government structure provides legal and care services. However, civil society has organized to address this contingency, and in Nuevo León there are organizations that 4.87 $\%$ are related to women's care in these cases. They perform care functions, human rights, self care, education and guidance, mental health services, capacity building and social reintegration.
\end{abstract}

\title{
Keywords
}

Civil society, non-governmental organizations, care, violence, gender, women

\section{Introducción}

En la actualidad, hay una gran presencia de agrupaciones, como también de organismos y organizaciones no gubernamentales (ONG) que han surgido tanto en los ámbitos internacionales como nacionales en distintos países. En este sentido comenta Gómez-Quintero (2014, p. 360), que este suceso "ha sido denominado por algunos como sociedad civil global [...] y obedece a una especie de revolución asociacional global [...] o a una verdadera explosión de la sociedad civil".

Las ONG se constituyen en lo que algunos autores como Pérez et al. (2011) han denominado como el Tercer Sector, que da completitud a la sociedad, ya que se estructura conjuntamente con los otros dos sectores que se reconocen como son el Estado y el Productivo. De tal manera que en el marco del siglo XXI estas entidades no gubernamentales son ahora un componente muy activo en el contexto social. 
Este florecimiento ha emergido societalmente, como Weber (1964) lo comenta, a partir de las relaciones interpersonales, de carácter individual o grupal, que tienen por concurrencia un propósito en común. Y que buscan, además, trascender mediante sus actividades, cuyo fin último es incidir sobre el contexto socioambiental en que subsisten (Escobar, 2010)

Por consiguiente, las ONG de acuerdo con Luciano Tomassini (citado por Pérez, 2010), permiten identificar como la sociedad civil se organiza - a la vez se configura en distintas agrupaciones y/o asociaciones - y que cada vez más toman un papel protagónico en los asuntos que conciernen e interesan a la comunidad. Por consiguiente, sus intervenciones tienen que ver también con aspectos que atañen - no solamente los de orden local o nacional, sino que además incurren sus actuaciones en asuntos del ámbito internacional.

Muchas son los campos de acción de estas ONG, entre los cuales atienden aspectos ambientales, en relación con los derechos humanos, problemáticas sociales, como también de orden asistencial y de salud, entre otras, es decir se dedican a una constelación de actividades muy variadas. Pero cuyas particularidades que destacan en todas las ONG son la subsidiariedad, la solidaridad, el apoyo desinteresado, como también el bien común.

En este marco antes comentado, esta investigación pretende caracterizar las ONG que dan fortaleza social y cuyo efecto incide en atender la problemática de violencia de género, en el contexto de la estructura comunitaria del Estado de Nuevo León, México.

\section{Marco conceptual}

En la actualidad la violencia de género ha sido una de las problemáticas sociales más relevantes y difíciles de atender, ya que esta tiene sus entrañas en los elementos socioculturales que han otorgado al varón la idea de una condición de superioridad que ha concebido una absurda asimetría social entre las mujeres y los hombres (Expósito, 2011). Esta heterosexualidad, en palabras de Suárez (2010), cuando se edifica mediante constructos e intereses particulares ya sean de orden social como de carácter cultural, origina una violencia que puede iniciar figuradamente para después mostrarse mediante hechos reales. 
¿Qué es la violencia? Particularmente en el marco de Declaración 48/104 promulgada por la Asamblea General de las Naciones Unidas sobre la Eliminación de la Violencia contra la Mujer, tipifica esta como:

Todo acto de violencia basado en la diferencia de género que tenga o pueda tener como resultado un daño o sufrimiento físico, sexual o psicológico para la mujer, inclusive las amenazas de tales actos, la coacción o la privación arbitraria de la libertad, tanto si se producen en la vida pública como privada. (Rico, 1996, p. 12)

Con esta postura de carácter internacional permitió en los últimos años del siglo pasado poner en evidencia la violencia ejercida contra las mujeres y esencialmente aquellas transgresiones a sus derechos humanos. Es así que la invisibilidad social de este suceso fue mostrada y la lamentable especificidad que tiene, tan solo por el hecho de ser mujer (Yugueros, 2014).

Durante este mismo período de tiempo, particularmente en 1993, la Organización Panamericana de la Salud (OPS) se pronunció - a través del Comité de Mujer, Salud y Desarrollo- que era extremadamente relevante y pertinente elaborar un plan de trabajo para atender la temática de la violencia contra las mujeres, por considerarlo como un problema de salud pública que aquejaba a la sociedad en general y a su vez invitaba a tomar acciones inmediatamente (Sagot \& Carcedo, 2000).

Esto sucedía, dada la prevalencia tan explícita que subsistía de la violencia contra la mujer, esencialmente porque constituía un hecho que al consumarse representaba un evento de invalidez o bien de fallecimiento entre las jóvenes y esposas en edad reproductiva. De tal manera que este conocimiento impulsó que las autoridades gubernamentales, como la sociedad civil se ocuparan en capacitar al personal de salud, y con ello se produjeran adicionalmente un fortalecimiento a los marcos legales y políticas públicas, esencialmente aquellas que incidían en el desarrollo social como también en las de protección a la mujer y a las niñas (Velzeboer et al., 2003).

La violencia establecida en el género, de acuerdo con Osborne (2009), cuenta con distintos factores que se muestran desde la opresión directa hasta un escenario de abuso y dominación de forma indirecta sobre la mujer. Es así, que Sagot y Carcedo (2000) hace mención, que aún sin importar cuan explícitamente se manifieste la violencia física contra la mujer, se debe recordar que son las consecuencias psicológicas las de más graves consecuencias por sus efectos prolongados en el tiempo. 
La Organización Mundial de la Salud (2017), señala textualmente en su publicación sobre datos y cifras relacionadas con la violencia de género algunas particularidades muy elocuentes al respecto, que indican que una tercera parte de las mujeres en el mundo - en algún momento de su vida - han padecido violencia, cuya expresión puede ser física y sexual, y esta tiene su génesis en su pareja o proviene de alguna otra persona ajena a ella. Asimismo, este organismo internacional agrega que $38 \%$ de los homicidios perpetrados en el orbe contra las mujeres proceden de su pareja masculina.

Estos acontecimientos, documentados internacionalmente contra las mujeres se constituyen en flagrantes acciones de exclusión y sometimiento social, tan solo por ser mujer, violando así de forma constante sus derechos humanos (Alvarado \& Guerra, 2012). Sin embargo, Vega et al. (2011) citan que aún la violencia basada en género puede surgir inclusive desde antes del nacimiento, cuando los padres y las madres en la búsqueda del nacimiento de un varón, pueden ejercer de manera coercitiva que las mujeres realicen abortos de manera selectiva.

\section{Metodología}

La metodología que se desarrolló para la presente revisión está en el marco de la catalogada como descriptiva y de carácter cualitativo, ya que se focaliza en una exploración documental de información tanto gubernamental como no gubernamental, para contestar las siguientes preguntas ¿Cuál es la problemática existente de violencia en contra de la mujer? Como también ¿Qué ONG inciden en atender esta contrariedad social y que clase de apoyo brindan en el Estado de Nuevo León? Para responder a estos cuestionamientos se indagó en fuentes primarias como secundarias que provienen del Sistema de Información Científica RedALyC, Biblat-Bibliografía Latinoamericana portal de las bases de datos CLASE y PERIÓDICA, Repositorio Académico Digital de la Universidad Autónoma de Nuevo León, del Instituto Nacional de Estadística y Geografía en México, del Instituto Estatal de las Mujeres en Nuevo León y Secretaria de Desarrollo Social del Estado de Nuevo León; llevando a cabo una búsqueda con los siguientes descriptores: violencia de género, México, Nuevo León, organizaciones civiles, organizaciones no gubernamentales. Posteriormente se realizó una lectura de los contenidos haciendo un análisis crítico que nos permitió acoger el objeto de 
estudio a tratar. En este trabajo se revisa el contexto nacional sucintamente y el caso de la Entidad Federativa de Nuevo León (México), para dar cuenta de la estructura civil organizada que atiende la problemática relativa a la violencia de género.

\section{Hallazgos}

\section{Situación en México}

De acuerdo con Casique $(2017$, p. 8) en México se han llevado a cabo esfuerzos notables por reconocer y prevenir esta problemática social relativa a la violencia contra la mujer, para ello se da cuenta de las firmas y adherencia a distintos acuerdos de orden internacional como:

La Primera Conferencia Internacional de la Mujer realizada en México en 1975, la Convención para la Eliminación de Todas las Formas de Violencia contra las Mujeres (CEDAW, por sus siglas en inglés) en 1979, la Cuarta Conferencia Internacional de la Mujer celebrada en Beijing en 1995 y la Convención Interamericana para Prevenir, Sancionar y Erradicar la Violencia Contra las Mujeres de Belem do Pará [en 1994].

Por ello, en México, se erigió la Ley General de Acceso de las Mujeres a una Vida Libre de Violencia en el año 2007, que en el artículo 5 del apartado IV establece que la violencia hacia las mujeres se tipifica jurídicamente como "cualquier acción u omisión, basada en su género, que les cause daño o sufrimiento psicológico, físico, patrimonial, económico, sexual o la muerte tanto en el ámbito privado como en el público".

No obstante, el marco legal que da cuenta de la violencia de género, en nuestro país de acuerdo con Ramírez (2015), este precepto jurídico no ha trascendido de manera suficiente en la conciencia social, no obstante que en el marco de las normas y herramientas de orden jurídico se han establecido argumentaciones que contrarrestan la discriminación por motivos de género. Esto prevalece debido a los estereotipos sociales que se han configurado en derredor de la figura femenina, al representarla con alto nivel de fidelidad, de abnegación, pero particularmente de obediencia e incluso de soportar la violencia contra su persona. Lo que significa que esta situación se ha 
erigido en un problema de orden público que ha incluido la muerte, lesiones y una desvalorización manifiesta de la calidad de vida tan solo por el hecho de ser mujer (Díaz, 2009).

En este sentido, de acuerdo con la Encuesta Nacional sobre la Dinámica de las Relaciones en los Hogares (ENDIREH) llevada a cabo por el Instituto Nacional de Estadística y Geografía-INEGI (2016), el 66.1 \% de las mujeres mexicanas de 15 años o más, han padecido al menos un acontecimiento de violencia, donde destaca la violencia emocional en el $49 \%$ de los casos, continuando la violencia sexual en el $41.3 \%$, posteriormente la violencia de orden físico en el $34 \%$ y finalmente la relativa a la discriminación económica o en el trabajo en el $29 \%$.

Además, documenta que la violencia de género no se circunscribe únicamente a un ámbito sino también ocurre en otros espacios sociales. Es así que en México durante el 2016 de acuerdo con la ENDIREH la prevalencia de violencia contra la mujer fue perpetrada en el $43.9 \%$ de las mujeres por su pareja, $38.7 \%$ de manera comunitaria, $26.6 \%$ en el sitio de trabajo, $25.3 \%$ en el ámbito escolar y $10.3 \%$ por algún familiar, reportando además que el $78.6 \%$ no solicito ningún apoyo a alguna institución o levantó una denuncia después de ser violentadas en su persona.

Por otra parte, Mellissa Galván en 2019, menciona en su investigación periodística datos que dan cuenta de la violencia en México contra las mujeres, al señalar que son asesinadas nueve mujeres diariamente en el país, por consiguiente, en el periodo de 2015 a 2019 se registraron 3200 feminicidios en el territorio nacional. A lo anterior agrega que Veracruz es el Estado donde han acontecido 104 feminicidios durante el primer semestre de 2019, por lo cual lo coloca como el más peligroso para las mujeres, siguiéndole el Estado de México con 42 casos, mientras que en la Cd. de México, en este mismo lapso de tiempo acumulaba ya 18 feminicidios.

Por otra parte, esta misma información periodística revela que, en el lapso de enero a agosto de 2019, 292 mujeres fueron violentadas sexualmente en la Cd. de México, donde $1.4 \%$ de esas fueron violaciones de orden tumultuaria. Este comportamiento viene precedido de una tasa de delitos sexuales - en 2017 - contra las mujeres que alcanzó la cifra de 2733 víctimas por cada 100000 mujeres.

Además, Galván (2019) menciona en su análisis que en un lapso de seis años - 2013 a 2018 - la percepción de la inseguridad por parte de las mujeres se incrementó en un $7.4 \%$ - de $74.7 \%$ a $82.1 \%$ - no importando si 
es un lugar público o privado, habiendo mencionado principalmente lugares como los cajeros bancarios automáticos $(87 \%)$, al abordar el transporte público $(74.2 \%)$, al transitar por la calle $(72.9 \%)$, entre otros. En tanto que la violencia de pareja proviene principalmente de su marido o exmarido, como también de los exprometidos de las mujeres, catalogando estas acciones como severas a muy severas en el $64 \%$ de los eventos. Aquí vale la pena mencionar que en México las mujeres de 15 años y más en un 19.4 $\%$ reportan haber tenido al menos un acto de violencia por sus parejas, que configuran agresiones físicas, estrangulamientos, abusos sexuales y amenazas con armas de fuego.

Igualmente, este estudio añade que es en el Estado de México, Cd. de México y la Entidad Federativa de Aguascalientes donde mayormente se ejerce la violencia de carácter emocional, económica, física y sexual en contra de las mujeres por parte de su pareja, contabilizando cifras del $53.3 \%$, $52.6 \%$ y $49.8 \%$-respectivamente - de los casos denunciados por las víctimas. Entre las consecuencias que emanan de estos actos, ha conllevado a documentar que en 2016 se perdieron 29.7 días de trabajo asalariado por cada mujer victimada. A la par de la información anteriormente mencionada, Galván (2019), cita que $33 \%$ de las mujeres arrestadas por parte de las corporaciones policiacas, han denunciado haber sido violentadas sexualmente por los miembros de estos grupos policiales y finalmente asevera que la violencia se exacerba cuando se trata de mujeres transgénero, lesbianas o bisexuales, ya que la constitución física de sus cuerpos no se apega a los rasgos de lo femenino.

Conforme a lo anterior, las ONG que atienden estas situaciones y otorgan servicios a las mujeres violentadas se han instaurado en la sociedad mexicana con la finalidad ejercer una actuación que influya y visibilice esta problemática social ante las instituciones gubernamentales. Si bien estas ONG no resuelven la complicación, si contribuyen a ocupar las ausencias de atención social por la falta de actividades y/o interés de las estructuras gubernamentales (Olvera, 2015).

\section{Caso Nuevo León}

La estructura sociodemográfica de las mujeres en el Estado de Nuevo León aglutina el $4.3 \%$ de las mujeres en el país, y estas representan el 50.3 
$\%$ de la población con la que cuenta esta entidad federativa, y el $37.3 \%$ de estas constituyen parte de la población económicamente activa (PEA). El nivel de escolaridad que ostentan las mujeres económicamente activas es de primaria no terminada $4 \%$, primaria terminada $14 \%$, secundaria completa el $51 \%$ y medio superior y superior constituyen el $31 \%$ (INEGI, 2015).

Las mujeres de la PEA de acuerdo con su condición conyugal $43 \%$ son casadas, $34 \%$ solteras, $9 \%$ se hallan en unión libre, $7 \%$ se encuentran separadas, $4 \%$ son viudas y $3 \%$ están divorciadas. En particular, las mujeres que se encuentran en la PEA, el $85.8 \%$ de ellas realizan además quehaceres domésticos, el $7.2 \%$ solo trabajan, el $5.4 \%$ adicionalmente estudian y el 1.6 $\%$ reportan dar a poyo al hogar. En relación con la jefatura de los hogares, el $24 \%$ de estos los encabezan las mujeres (INEGI, 2015).

Sin embargo, el Estado de Nuevo León, tal como sucede en el escenario nacional, cuenta con un comportamiento muy similar, y de acuerdo con la ENDIREH (INEGI, 2016) la violencia contra la mujer nuevoleonesa fue reportada en el $59.3 \%$. El $35.7 \%$ fue consumada de manera comunitaria, el $32.2 \%$ por su pareja, $23 \%$ en el espacio de trabajo y $18.4 \%$ en el ámbito escolar. En esta misma línea discursiva podemos agregar también que la tendencia con respecto a la prevalencia de victimización en mujeres en Nuevo León se ha incrementado, concibiendo como victimización un delito que afecta a una mujer. En 2010 se contaba con 22899 casos por cada cien mil habitantes y en 2018 se exhibe 24553 casos por cada cien mil habitantes, es decir aumentó en un $6.7 \%$ (INEGI, 2019).

Por ello, actualmente el Estado de Nuevo León, a través de su gobierno, cuenta con una red administrativa que brinda tanto atención y orientación sin ningún cargo económico a las mujeres que se encuentran en un escenario de violencia (Gobierno de Nuevo León, 2019). Entre los servicios que otorgan se encuentra la asesoría legal, la atención psicológica y de asistencia social, por medio de agencias de ministerio público, centros de justicia familiar, centros de atención y prevención a la violencia familiar, centros de atención familiar y mediante el Instituto Estatal de las Mujeres.

En este caso, destacamos la instancia del Instituto Estatal de las Mujeres (2019), que cuenta en el marco de sus responsabilidades el de promover políticas públicas que coadyuven y garanticen acciones que estriben en la no discriminación y exclusión social de las mujeres, y además afirmen el respeto íntegro de los derechos humanos de las mujeres. Otro rasgo de esta estructura administrativa de carácter gubernamental, es que impulsan la Alerta de Vio- 
lencia de Género contra las Mujeres (AVGM), que es el dispositivo determinado en la Ley General de Acceso de las Mujeres a una Vida Libre de Violencia, para garantizar la seguridad jurídica tanto de mujeres como niñas.

No obstante, lo anterior, la sociedad civil al observar la problemática vigente, se ha organizado de manera estructurada para apoyar, en una genuina acción de gobernanza, la atención a mujeres que han sido violentadas y coadyuvar en el apoyo social que se requiere de manera pertinente en estos casos. Es necesario recalcar, que la gobernanza la concebimos como aquella interacción que surge entre las entidades gubernamentales y la sociedad civil para atender las problemáticas sociales (Canto, 2008). De esta manera, las ONG suscritas en el padrón de organizaciones civiles del Gobierno del Estado de Nuevo León durante el 2019 y que están enfocadas particularmente al desarrollo social fueron 595 agrupaciones; de las cuales $4.87 \%$ ( $\mathrm{f}=29)$ están relacionadas con la atención a la mujer.

Pudimos caracterizar a estas 29 ONG luego de una revisión de sus propósitos y de acuerdo con sus fines en ocho rutas que acogen fundamentalmente. A continuación, hacemos alusión a estas líneas de acción detectadas en el marco de sus pronunciamientos por parte de las ONG:

- Atención de mujeres sujetas a violencia: promueven la prevención, protección y otorgan servicios multidisciplinarios en mujeres que coexisten bajo el yugo de la violencia familiar. Representan el 24.1 $\%$ de las instituciones y encontramos a las ONG: Alternativas Pacíficas, A.C.; Asociación de Salud Integral y Desarrollo Personal, A.C.; Centro de Investigación Familiar, A.C.; Explora-T, A.C.; Instituto de Mujer Restaurada, A.C.; Líderes con Visión de Ayudar y Transformar, A.C.; Mujeres de Valor, A.B.P.; 7) Viccali, A.C.

- Atienden los derechos humanos de las mujeres: llevan a cabo un análisis de los marcos legales vigentes y de las políticas públicas con la finalidad de hacer propuestas de solución, en el marco de los derechos humanos, a este fenómeno social. Personifican el $17.2 \%$ de las instituciones y hallamos a las ONG como la 1) Agrupación Política Femenina, A.C.; Arthemisas por la Equidad, A.C.; 3) Asociación de Sororidad Tanatológica Ana Sulivan, A.C.; Mariposas de la Felicidad, A.C.; Mujer en Plenitud, A.B.P.

- Promueven entre las mujeres el autocuidado: proveen orientación para concientizar a las mujeres en su autocuidado en aspectos mé- 
dicos, legales y psicológicos y así afrontar las dificultades de un entorno violento, buscando que estas preserven su dignidad y así fortalecer el empoderamiento sobre su persona. Constituyen el $13.7 \%$ de las instituciones y localizamos a. Asociación Mujer Saludable, A.C.; Centro de Ayuda para la Mujer Latinoamericana, A.C.; Centro de Orientación Familiar Jahdiel, A.C.; Ellas en Movimiento, A.C.

- Intervienen socialmente con programas de educación y orientación en las mujeres: concientizan y educan a las mujeres sobre los aspectos que inmiscuye la violencia de género en el marco de hacer florecer de nueva cuenta la dignidad en las personas. Componen el 13.7 $\%$ de las instituciones y advertimos: Grupo Interdisciplinario para Asuntos de la Mujer, A.C.; Sendas de Victoria, A.C.; 3) Tranvida A.C.; Tejedoras de Cambio, A.C.

- Otorgan servicios de salud mental a las mujeres violentadas: brindan atención terapéutica para frenar los traumas y ciclos de sufrimiento provocados por la violencia en las mujeres. Forman el $10.3 \%$ de las instituciones y observamos a la Asociación Mexicana para Ayuda Mental en Crisis, A.C.; Asociación Psicoanalítica de Orientación Lancaniana, A.C.; Asociación Regiomontana de Psicoanálisis, A.C.

- Ofrecen formación y desarrollo de capacidades a las mujeres: apoyan a las mujeres sujetas a violencia mediante una oportunidad de superación técnico profesional, para que puedan independizarse y retomen un nuevo proyecto de vida. Configuran el 10.3\% de las instituciones y nos percatamos del Instituto de Educación Preescolar, A.C.; Centro Pedagógico Cumbres; Promesa Femenina, A.C.

- Asistencia social a las mujeres que sufren de violencia: contribuyen mediante labores asistenciales a sufragar las carencias de las condiciones de vida en que subsisten las mujeres como sus familias también. Plasman el $6.8 \%$ de las instituciones y encontramos a Vida y Familia Monterrey, A.C.; Mujeres Que Saben Creer, A.C.

- La reinserción social de las mujeres agredidas: ofrecen un espacio de hospedaje y alimentación que permite que las mujeres que han sido sujetas a violencia, puedan retomar el control de sus personas y se integren paulatinamente a la vida social y productiva. Materializan el $3.4 \%$ de las instituciones y hallamos a la Fundación Lamentos Escuchados, A.C. 
Como se evidencia en las ONG se consolida la red social que permite a las mujeres sujetas de violencia contar con protección mediante el marco de los derechos humanos, donde se les educa y orienta para su autocuidado. Pero, además, reciben servicios de salud mental con la finalidad de restablecer su dignidad y competencias sociales, recibiendo asistencia social durante estos periodos y finalmente tener una nueva oportunidad de reinsertarse en el núcleo social, con el fin de recuperar el curso de su vida.

\section{Discusión}

Durante este análisis nos hemos podido percatar de la relación que subsiste en Nuevo León entre el gobierno con la sociedad, la cual favorece la participación ciudadana, que fortalece por una parte la intervención de esta en los procesos y situaciones sociales que implican una atención. Se debe agregar, que de esta forma la sociedad civil de manera organizada incide en las estructuras administrativas de orden gubernamental y por consecuencia en la aplicación de las políticas públicas (Quintero-Castellanos, 2017).

Por otra parte, las ONG incurren de acuerdo con sus propósitos y misiones trazadas en una lectura que hacen de su entorno socioambiental, que les permite a estas detectar asuntos colectivos que quebrantan o satisfacen el orden social y gubernamental, y sus actuaciones se centralizan para modificar o sostener el contexto social prevaleciente, en el marco que les asiste como partícipes activos de una sociedad democrática, de reconocimiento y respeto a los derechos de las personas. De esta manera, se insertan en el ámbito de la decisiones y actuaciones públicas (Canto, 2008).

Es así que las ONG que atienden en Nuevo León la problemática y atención a la mujer que subsiste en un entorno de violencia, está demarcado desde la perspectiva de la gobernanza por organizaciones civiles estructuradas y por una comprensión de la problemática existente, en la que se muestran expresiones de un ejercicio poder público (Aguilar, 2011). Adicionalmente esto pone a relieve una interacción de orden horizontal entre las dependencias públicas, las instituciones privadas y la comunidad (Brower, 2016).

Si bien la gobernanza explícitamente vista a través de las ONG puede surgir en un ambiente caracterizado por las demandas y tensiones por la vinculación de estas con las realidades de la sociedad, como es en este caso la atención a la violencia en que subsisten las mujeres, se debe hacer hincapié 
que las actuaciones que emergen de forma organizada en la sociedad civil siempre están vinculadas a los grandes acuerdos sociales que están plasmados en las políticas públicas y en el desempeño de los gobiernos (De Sousa Santos, 2004).

Desde nuestra posición de análisis, las ONG de manera general, pero en particular al objeto de estudio que estamos analizando que trata sobre las agrupaciones que ayudan en la atención a la mujer que es sujeta de violencia en Nuevo León, estas organizaciones se levantan como actores que articulan legítimamente las necesidades de la sociedad emanadas de esta problemática y las aúnan a las pautas de solución de orden gubernamental, con el propósito de impactar socialmente en el ámbito cultural como en la parte estructural de la sociedad, esto es sensibilizando e interviniendo en el colectivo social, donde tiene su génesis la violencia producto de las interacciones sociales, y donde además se experimenta y transcribe en el tejido social (Martínez, 2008).

\section{Bibliografía}

Aguilar, L. F. (2011). Gobernanza pública para obtener resultados: marco conceptual y operacional. Organización de las Naciones Unidas.

Alvarado, M., \& Guerra, N. (2012). La violencia de género un problema de salud pública. Interacción y Perspectiva, Revista de Trabajo Social, 2(2), 117130 .

Brower, J. (2016). En torno al sentido de gobernabilidad y gobernanza: delimitación y alcances. Daimon. Revista Internacional de Filosofía, 67, 149-162. https://doi.org/10.6018/202011

Canto, M. (2008). Gobernanza y participación ciudadana en las políticas públicas frente al reto del desarrollo. Política y Cultura, 30, 9-37. https://bit. ly/3cOLmbb

Casique, I. (2017). Fuentes y datos sobre la violencia contra las mujeres en México. Aprendizajes, dificultades y retos acumulados. Revista Internacional de Estadística y Geografía, 8(1), 6-16. https://bit.ly/3gzSqe7

De Sousa Santos, B. (2004). Democratizar la Democracia: los caminos de la democracia participativa. Fondo de Cultura Económica.

Díaz, G. (2009). La violencia de género en México: reto del gobierno y de la sociedad. Encrucijada. Revista Electrónica del Centro de Estudios en Ad- 
ministración Pública, 2 (mayo-agosto), 1-16.http://dx.doi.org/10.22201/ fcpys.20071949e.2009.2.58551

Escobar, R. A. (2010). Las ONG como organizaciones sociales y agentes de transformación de la realidad: desarrollo histórico, evolución y clasificación. Diálogos de Saberes, 32, 121-131. https://bit.ly/3xvzL8I

Expósito, F. (2011). Violencia de género. Mente y Cerebro, 48, 20-25.

Galván, M. (19 agosto 2019). 14 datos de la violencia de género que explican el enojo de las mujeres. Expansión Política. https://bit.ly/3iOD7zD

Gobierno del Estado de Nuevo León (2019). Centros y módulos de atención para mujeres en situación de violencia. https://bit.ly/35uagJ2

Gobierno del Estado de Nuevo León (2019). Directorio de Organizaciones de la Sociedad Civil en el Estado de Nuevo León enfocadas al Desarrollo Social. Monterrey: Secretaría de Desarrollo Social-Gobierno de Estado de Nuevo León.

Gómez-Quintero, J. D. (2014). Organizaciones no gubernamentales y entidades sin ánimo de lucro en Colombia. Despolitización de la sociedad civil y tercerización del Estado. Gestión y Política Pública, 23(2), 359-384. https:// bit.ly/3gG66D8

Instituto Estatal de las Mujeres (2019). Alerta de violencia de género contra las mujeres. https://bit.ly/3zAhjh5

Instituto Nacional de Estadística y Geografía (INEGI) (2015). Encuesta Intercensal-2015. https://bit.ly/3gxbUjt

Instituto Nacional de Estadística y Geografía (INEGI) (2016). ENDIREH (Encuesta Nacional sobre la Dinámica de las Relaciones en los Hogares)-2016. INEGI. https://bit.ly/3vuTggk

Instituto Nacional de Estadística y Geografía (INEGI) (2019). Seguridad pública y justicia-victimización. https://bit.ly/2U5G9VO

Ley General de Acceso de las Mujeres a una Vida Libre de Violencia (2007). Publicada en el Diario Oficial de la Federación el 1 de febrero de 2007. México: Estados Unidos Mexicanos.

Martínez, A. (2008). Breve cartografía del estudio sobre la violencia. (Tesis de Licenciatura). Universidad Autónoma Metropolitana-Azcapotzalco. Cd. de México, México.

Olvera, A. I. (2015). Representaciones e ideologías de los organismos civiles en México: crítica de la selectividad y rescate del sentido de la idea de sociedad civil. En J. Cadena (Coord.), Las organizaciones civiles mexicanas hoy (pp. 23-47). UNAM. 
Organización Mundial de la Salud (2017). Violencia contra la mujer. https://bit. ly/3cRgTcp

Osborne, R. (2009). Apuntes sobre violencia de género. Ediciones Bellaterra.

Pérez, G., Arango, M. D., \& Sepulveda, L. Y. (2011). Las organizaciones no gubernamentales ONG: hacia la construcción de su significado. Ensayos de Economía, 38 (enero-junio), 243-260. https://bit.ly/3gyPnTm

Pérez, M. (2010). Organizaciones no gubernamentales, características de otro actor en el tablero internacional. Centro Argentino de Estudios Internacionales.

Quintero, C. E. (2017). Gobernanza y teoría de las organizaciones. Perfiles Latinoamericanos, 25(50), 39-57. https://bit.ly/3wATtQD

Ramírez, G. (2015). La violencia de género, un obstáculo a la igualdad. Trabajo Social UNAM, 10, 43-58. https://bit.ly/3iMmiVV

Rico, N. (1996). Violencia de género: un problema de derechos humanos. Serie Mujer y Desarrollo No. 16. Comisión Económica para América Latina y el Caribe.

Sagot, M., \& Carcedo, A. (2000). Ruta crítica de las mujeres afectadas por la violencia intrafamiliar en América Latina. Estudios de caso en diez países. Organización Panamericana de la Salud.

Suárez, M. (2010). Derecho, violencia y género en el México de los albores del siglo XXI. Revista Fuentes humanísticas, 22(41), 109-122. https://bit. ly/3zCW $1 z 1$

Vega, G., Hidalgo, D., \& Toro, J. (2011). Violencia basada en género desde la perspectiva médica. Revista de Obstetricia y Ginecología de Venezuela, 71(2), 88-97. https://bit.ly/35yu5PA

Velzeboer, M., Ellsberg, M., Clave-Arcas, C., \& García-Moreno, C. (2003). La violencia contra las mujeres: responde el sector salud. Organización Panamericana de la Salud.

Weber, M. (1964). Economía y Sociedad. Fondo de Cultura Económica.

Yugueros, A.J. (2014). La violencia contra las mujeres: conceptos y causas. Barataria Revista Castellano-Manchega de Ciencias Sociales, 18, 147-159. https://bit.ly/3cPUtbA 